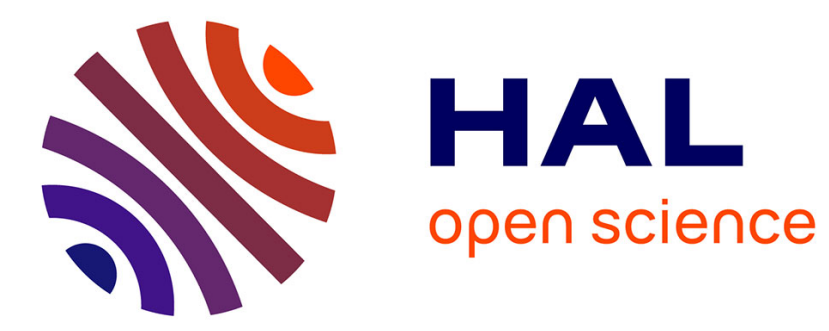

\title{
A sharp contact discontinuity scheme for multimaterial models
}

\author{
Angelo Iollo, Thomas Milcent, Haysam Telib
}

\section{To cite this version:}

Angelo Iollo, Thomas Milcent, Haysam Telib. A sharp contact discontinuity scheme for multimaterial models. [Research Report] RR-7534, INRIA. 2011, pp.25. inria-00565339

\section{HAL Id: inria-00565339 \\ https://hal.inria.fr/inria-00565339}

Submitted on 13 Feb 2011

HAL is a multi-disciplinary open access archive for the deposit and dissemination of scientific research documents, whether they are published or not. The documents may come from teaching and research institutions in France or abroad, or from public or private research centers.
L'archive ouverte pluridisciplinaire HAL, est destinée au dépôt et à la diffusion de documents scientifiques de niveau recherche, publiés ou non, émanant des établissements d'enseignement et de recherche français ou étrangers, des laboratoires publics ou privés. 
INSTITUT NATIONAL DE RECHERCHE EN INFORMATIQUE ET EN AUTOMATIQUE

\title{
A sharp contact discontinuity scheme for multimaterial models
}

\author{
Angelo Iollo — Thomas Milcent — Haysam Telib
}

\section{$\mathbf{N}^{\circ} \mathbf{7 5 3 4}$}

Février 2011

Computational models and simulation

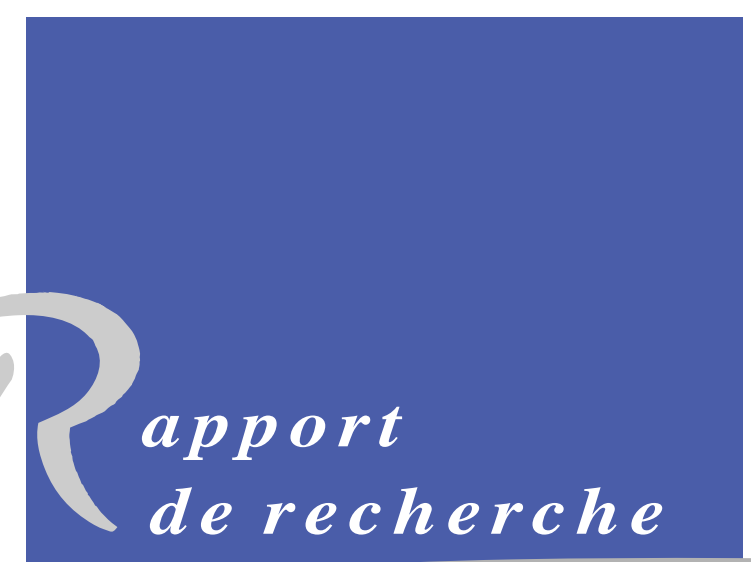





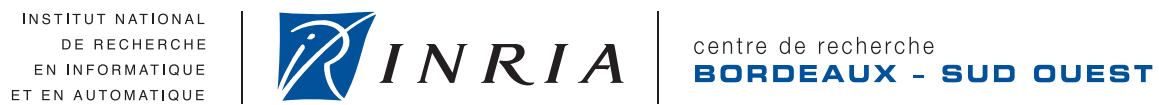

\title{
A sharp contact discontinuity scheme for multimaterial models
}

\author{
Angelo Iollo*, Thomas Milcent* , Haysam Telibむ \\ Theme : Computational models and simulation \\ Applied Mathematics, Computation and Simulation \\ Équipe-Projet MC2
}

Rapport de recherche $n^{\circ} 7534-$ Février $2011-22$ pages

\begin{abstract}
We present a method to capture the evolution of a contact discontinuity separating two different materials. This method builds on the ghost-fluid idea: a locally non-conservative scheme allows an accurate and stable simulation of problems involving non-miscible media that have significantly different physical properties. Compared to the ghost-fluid approach, the main difference is that with the present method the contact discontinuity stays sharp. Numerical illustrations ranging from air-water interactions to non-linear elasticity are presented.
\end{abstract}

Key-words: Multimaterial scheme, sharp interface, ghost-fluid

* INRIA Bordeaux Sud-Ouest, Equipe MC2

$\dagger$ Dipartimento di Ingegneria Aeronautica e Spaziale, Politecnico di Torino

Centre de recherche INRIA Bordeaux - Sud Ouest 


\section{Un schéma "sharp interface" pour des modèles multimatériaux}

Résumé : Nous présentons une méthode pour capturer l'évolution d'une discontinuité de contact séparant deux matériaux différents. Cette méthode repose sur l'idée "ghost-fluid": un schéma localement non-conservatif permet une simulation précise et stable de problèmes impliquant des milieux non-miscibles qui ont des propriétés physiques différentes. La principale différence avec l'approche "ghost-fluid" est que cette méthode traite de manière "sharp" la discontinuité de contact. Des illustrations numériques allant des interactions air-eau à l'élasticité non-linéaire sont présentées.

Mots-clés : schémas multimatériaux, interface raide, "ghost-fluid" 


\section{Introduction}

Physical and engineering problems that involve several materials are ubiquitous in nature and in applications: multiphase flows, fluid-structure interaction in aeronautical, bio-medical and ocean engineering, to cite just a few examples. Many different strategies were proposed to attack this problem in the literature: lagrangian schemes (see for example [11]), arbitrary-lagrangian-eulerian (ALE) schemes ([7] for a recent application), and eulerian schemes (as in [14]). The literature in this domain is vast and each of the approaches cited include a variety of specific methods. Broadly speaking lagrangian methods are interesting because the interface between the material is fixed in the reference domain and the interface conditions can be taken into account in a simple and accurate way. However these methods suffers from the fact that if one considers fluid flows, then the mapping from the physical to the reference domain can become very irregular since the distance between material points that are close in the reference domain may become large after a finite time. ALE methods take into account this peculiarity of fluid flows by using a discretization in the physical domain over an unsteady mesh that copes with the moving interfaces, thus allowing an accurate discretization of both the fluid flow and the interface conditions. The counterpart is the complexity of the scheme formulation and of the numerical implementation. Finally, eulerian schemes discretize the model in the physical plane on a fixed mesh, but additional care is required to accurately and consistently describe interface conditions. In this paper we are interested in eulerian models describing phenomena such as the interaction of a shock wave at a gas/water interface, or the propagation of non-linear elastic waves from a solid to a fluid, by using a single set of equations.

The main contributions in the direction of simulating these phenomena go back to 9 and 12 for the model and to 2 for a consistent and stable discretization. The idea is to model the eulerian stress tensor through a constitutive law reproducing the mechanical characteristics of the medium under consideration. Hence, for example, an elastic material or a gas will be modeled by the same set of equations except for the constitutive law relating the deformation and the stress tensor. The system of conservation laws thus obtained can be cast in the framework of quasi-linear hyperbolic partial differential equations (PDEs). From the numerical view point this is convenient since classical integration schemes can be employed in each material. However, it turns out that the evolution of the interface, which is represented in this model by a contact discontinuity, is particularly delicate because standard Godunov schemes fail. In 11 it was shown that a simple and effective remedy to this problem is the definition of a ghost fluid across the interface. A remarkable application based on this approach is presented in [8]. This method, however, has the disadvantage that the interface is diffused over a certain number of grid points. From a practical view point this can be a serious disadvantage if one is interested in the geometric properties of the evolving interface, as, for example, in the case of surface tension or when the interface itself is elastic. One way to approach this problem is through immersed boundary methods where using appropriately defined cut cells (see for example [10]) it is possible to keep the interface sharp. This is done at the cost of a rather sophisticated local analysis of the space-time evolution of the interface intersection with the grid. 
In this paper we propose a simple method to recover a sharp interface description keeping the solution stable and non-oscillating. The point of departure is a finite-volume discretization and a dimensional splitting. Then, the interface is treated like a contact discontinuity relative to the solution of an appropriate Riemann problem. In the following we detail the eulerian model used, the numerical method, and present a set of standard test-cases in one space dimension.

\section{The model}

The following model was described before in [9], [12], [5] and [8]. We report here the principal elements of the formulation and some further developments for the characteristic speeds.

The starting point is classical continuum mechanics. Let $\Omega_{0}$ be the reference or initial configuration of a single material and $\Omega_{t}$ the deformed configuration at time $t$. We define $X(\xi, t)$ as the image at time $t$ of a material point $\xi$ belonging to the initial configuration, in the deformed configuration:

$$
\begin{aligned}
X: \Omega_{0} \times[0, T] & \longrightarrow \Omega_{t} \\
(\xi, t) & \mapsto X(\xi, t)
\end{aligned}
$$

and the corresponding velocity field $u$ as

$$
\begin{aligned}
u: \Omega_{t} \times[0, T] & \longrightarrow \mathbb{R}^{3} \\
(x, t) & \mapsto u(x, t)
\end{aligned}
$$

where

$$
X_{t}(\xi, t)=u(X(\xi, t), t)
$$

completed by the initial condition $X(\xi, 0)=\xi$. Also we introduce the backward characteristics $Y(x, t)$ that for a time $t$ and a point $x$ in the deformed configuration, gives the corresponding initial point $\xi$ in the initial configuration, i.e.,

$$
\begin{aligned}
Y: \Omega_{t} \times[0, T] & \longrightarrow \Omega_{0} \\
(x, t) & \mapsto Y(x, t)
\end{aligned}
$$

with the initial condition $Y(x, 0)=x$. Of course, we have

$$
X(Y(x, t), t)=x \quad Y(X(\xi, t), t)=\xi .
$$

Differentiating with respect to time and space in turn, we obtain

$$
Y_{t}+(u \cdot \nabla) Y=0
$$

and

$$
\left[\nabla_{\xi} X(\xi, t)\right]=\left[\nabla_{x} Y(x, t)\right]^{-1}
$$

We are now in the position of writing the mass, momentum and energy conservation with respect to both the reference and the deformed configuration. 


\subsection{Lagrangian formulation}

In the reference domain $\Omega_{0}$ the governing equations can be written as a second order system of PDEs for $X(\xi, t)$ and $W(\xi, t)$, the internal energy per unit volume. In the reference domain, Newton's law and energy conservation are:

$$
\left\{\begin{aligned}
\rho_{0} X_{t t} & =\operatorname{div}_{\xi} \mathcal{T} \\
W_{t} & =\mathcal{T}: \nabla_{\xi} X_{t}
\end{aligned}\right.
$$

where $\mathcal{T}(\xi, t)$ is the first Piola-Kirchhoff stress tensor relative to the constitutive model of the material under consideration.

Given initial conditions for position, velocity, energy and the initial density distribution $\rho_{0}(\xi)$, these equations can be integrated in time to determine the deformed configuration. The trajectory $X(\Gamma, t)$ of an interface $\Gamma \subset \mathbb{R}^{3}$ between two different materials is described by this formulation. At this interface $X_{t}(\Gamma, t)$ is continuous, as well as the normal component of the stress tensor to grant equilibrium.

In elasticity, the internal energy is a function of the strain tensor $\nabla_{\xi} X$ and the entropy $s, W=W\left(\nabla_{\xi} X, s\right)$. The Piola-Kirchhoff stress tensor can be written as the derivative of a potential $W$ that has to be Galilean invariant and, eventually, isotropic. It can be proved that (Rivlin-Eriksen theorem [4) this is the case if, and only if, $\mathcal{E}$, the energy, is expressed as

$$
\mathcal{E}(t)=\int_{\Omega_{0}} \tilde{W}(\operatorname{Tr}(C(\xi, t)), \operatorname{Tr}(\operatorname{Cof}(C(\xi, t))), J(\xi, t), s(\xi, t)) d \xi
$$

where

$$
C(\xi, t)=\left[\nabla_{\xi} X\right]^{T}\left[\nabla_{\xi} X\right]
$$

is the right Cauchy-Green tensor and

$$
J(\xi, t)=\operatorname{det}\left(\left[\nabla_{\xi} X\right]\right)=\operatorname{det}(C)^{\frac{1}{2}} .
$$

We assume that the internal energy per unit volume is the sum of a term depending on volume variation and entropy, and a term accounting for isochoric deformation:

$$
\mathcal{E}(t)=\int_{\Omega_{0}}\left(W_{\text {vol }}(J, s)+W_{\text {iso }}(\operatorname{Tr}(\bar{C}), \operatorname{Tr}(\operatorname{Cof}(\bar{C})))\right) d \xi
$$

where

$$
\bar{C}(\xi, t)=\frac{C}{\operatorname{det}(C)^{\frac{1}{3}}} .
$$

In general the term relative to an isochoric transformation will also depend on entropy. Here, we will limit the discussion to materials where shear forces are conservative.

For an isoentropic transformation we have that $W_{t}=W^{\prime}\left(\nabla_{\xi} X, s\right): \nabla_{\xi} X_{t}$, with the prime denoting differentiation with respect to the first argument. 
Hence, from the energy equation (5) we have that $W^{\prime}\left(\nabla_{\xi} X, s\right)=\mathcal{T}\left(\nabla_{\xi} X, s\right)=$ $\nabla_{\xi} X \Sigma$, where

$$
\Sigma(\xi, t)=J W_{\mathrm{vol}}^{\prime} C^{-1}+2 J^{-\frac{2}{3}}\left(\bar{\Sigma}_{\text {iso }}-\frac{1}{3} C^{-1}\left(C: \bar{\Sigma}_{\text {iso }}\right)\right)
$$

is the second Piola-Kirchhoff stress tensor with

$$
\bar{\Sigma}_{\text {iso }}=\left(\frac{\partial W_{\text {iso }}}{\partial a}+\operatorname{Tr}(\bar{C}) \frac{\partial W_{\text {iso }}}{\partial b}\right) I-\frac{\partial W_{\text {iso }}}{\partial b} \bar{C}
$$

and where $\frac{\partial W_{\text {iso }}}{\partial a}$ and $\frac{\partial W_{\text {iso }}}{\partial b}$ are the partial derivatives of $W_{\text {iso }}(\operatorname{Tr}(\bar{C}), \operatorname{Tr}(\operatorname{Cof}(\bar{C})))$ with respect to its first and second argument, in turn.

\subsection{Eulerian formulation}

The counterpart of the above equations in the deformed configuration is:

$$
\left\{\begin{aligned}
\rho_{t}+\operatorname{div}_{x}(\rho u) & =0 \\
\rho\left(u_{t}+\left(u \cdot \nabla_{x}\right) u\right)-\operatorname{div}_{x} \sigma & =0 \\
\rho\left(\varepsilon_{t}+\left(u \cdot \nabla_{x}\right) \varepsilon\right)-\sigma: \nabla_{x} u & =0 \\
Y_{t}+\left(u \cdot \nabla_{x}\right) Y & =0
\end{aligned}\right.
$$

where $\sigma(x, t)$ is the Cauchy stress tensor in the physical domain. The unknowns are the backward characteristics of the problem $Y(x, t)$, the velocity $u(x, t)$, the internal energy per unit mass $\varepsilon(x, t)=W / \rho_{0}$ and the density $\rho(x, t)$. The initial velocity $u(x, 0)$, the initial internal energy $\varepsilon(x, 0)$ and $Y(x, 0)=x$ are given. If the initial density $\rho_{0}(\xi)$ is known, the equation of mass conservation is actually redundant because $\rho(x, t)=\operatorname{det}\left(\nabla_{x} Y(x, t)\right) \rho_{0}(Y(x, t))$.

To close the system, a constitutive law which connects $\sigma$ to $Y$ is necessary. The change of variables $\xi=Y(x, t)$ in the energy (7) gives with (4) (the invariants of $B$ and $C$ are the same)

$$
\mathcal{E}=\int_{\Omega_{t}}\left(W_{\text {vol }}(J, s)+W_{\text {iso }}(\operatorname{Tr}(\bar{B}), \operatorname{Tr}(\operatorname{Cof}(\bar{B}))) J^{-1} d x\right.
$$

where

$$
\bar{B}(x, t)=\frac{B(x, t)}{\operatorname{det}(B(x, t))^{\frac{1}{3}}} \quad B(x, t)=\left[\nabla_{x} Y(x, t)\right]^{-1}\left[\nabla_{x} Y(x, t)\right]^{-T}
$$

with $B(x, t)$ the left Cauchy-Green tensor and

$$
J(x, t)=\operatorname{det}\left(\left[\nabla_{x} Y(x, t)\right]\right)^{-1}=\operatorname{det}(B(x, t))^{\frac{1}{2}} .
$$

The second Piola-Kirchhoff stress tensor $\Sigma$ and the Cauchy stress tensor $\sigma$ are related by

$$
\sigma(x, t)=\operatorname{det}\left(\left[\nabla_{x} Y(x, t)\right]\right)\left[\nabla_{x} Y(x, t)\right]^{-1} \Sigma(Y(x, t), t)\left[\nabla_{x} Y(x, t)\right]^{-T} .
$$

Following (8) we get 


$$
\sigma(x, t)=W_{\mathrm{vol}}^{\prime}(J, s) I+2 J^{-1}\left(\bar{\sigma}_{\text {iso }}-\frac{1}{3} I\left(\bar{\sigma}_{\text {iso }}: I\right)\right)
$$

with

$$
\bar{\sigma}_{\text {iso }}=\frac{\partial W_{\text {iso }}}{\partial a} \bar{B}-\frac{\partial W_{\text {iso }}}{\partial b} \bar{B}^{-1}
$$

By definition pressure is given by $p=-\frac{1}{3} \operatorname{Tr}(\sigma)=-W_{\text {vol }}^{\prime}(J, s)$. This definition is coherent with the thermodynamic definition of pressure. Indeed, recalling that $\rho J=\rho_{0}$, we have $-W_{\mathrm{vol}}^{\prime}(J(\rho), s)=\frac{\rho^{2}}{\rho_{0}} \frac{\partial W(\rho, s)}{\partial \rho}=\rho^{2} \frac{\partial \varepsilon(\rho, s)}{\partial \rho}$.

We can put (9) in conservative form obtaining:

$$
\left\{\begin{aligned}
\rho_{t}+\operatorname{div}_{x}(\rho u) & =0 \\
(\rho u)_{t}+\operatorname{div}_{x}\left(\rho u \otimes u-\sigma\left(\nabla_{x} Y\right)\right) & =0 \\
(\rho e)_{t}+\operatorname{div}_{x}(\rho e u-\sigma u) & =0 \\
\left(\nabla_{x} Y\right)_{t}+\nabla_{x}\left(\left(u \cdot \nabla_{x}\right) Y\right) & =0
\end{aligned}\right.
$$

where $e(x, t)=\varepsilon+|u|^{2} / 2$ is the total energy per unit mass. It is this form that will be used in subsequent chapters.

\section{Characteristic speeds}

The models above encompass a large class of materials, thermodynamic transformations and cinematic patterns. We focus in the following on isoentropic deformations and we compute the characteristic speeds for models resulting from this hypothesis. The constitutive laws that we consider are conservative and hence entropy is always constant unless the solution becomes discontinuous. Under the condition $\frac{\partial W}{\partial s}=0$, we can discard the energy equation since the stress tensor will depend exclusively on the local deformation and hence in (10) energy equation is decoupled from the others.

For the objectives of this paper, we further restrict our investigation to a one-dimensional case with non-zero transverse velocity. Let $x_{i}, i=1 \ldots 2$ be the coordinates in the canonical basis of $\mathbb{R}^{2}, u_{i}$ the velocity components, $Y^{i}$ the components of $Y$ and $\sigma^{i j}$ the components of the stress tensor. Also, let us denote by $\cdot, i$ differentiation with respect to $x_{i}$. We consider the governing equations in two space dimensions and we assume that $\nabla Y$ is a function only of one direction $\left(x_{1}\right)$, as well as $u_{1}$ and $u_{2}$. In this case we have that $\left(Y_{, 2}^{1}\right)_{t}=\left(Y_{2}^{2}\right)_{t}=0$. Since $Y(x, 0)=x$, we have also that $Y_{, 2}^{1}=0, Y_{, 2}^{2}=1$ and hence

$$
[\nabla Y]=\left(\begin{array}{cc}
Y_{, 1}^{1} & 0 \\
Y_{, 1}^{2} & 1
\end{array}\right)
$$

The governing equations can then be written as

$$
\Psi_{t}+(F(\Psi))_{, 1}=0
$$

with 


$$
\Psi=\left(\begin{array}{c}
\rho \\
\phi_{1} \\
\phi_{2} \\
Y_{, 1}^{1} \\
Y_{, 1}^{2}
\end{array}\right) \quad F(\Psi)=\left(\begin{array}{c}
\phi_{1} \\
\frac{\left(\phi_{1}\right)^{2}}{\rho}-\sigma^{11} \\
\frac{\phi_{1} \phi_{2}}{\rho}-\sigma^{21} \\
\frac{\phi_{1} Y_{, 1}^{1}}{\rho} \\
\frac{\phi_{1} Y_{, 1}^{2}+\phi_{2}}{\rho}
\end{array}\right)
$$

and $\phi_{i}=\rho u_{i}$. The stress tensor $\sigma$ has only two non-zero components, hence we can simplify the notation by taking

$$
\sigma=\left(\begin{array}{l}
\sigma^{11}\left(Y_{, 1}^{1}, Y_{, 1}^{2}\right) \\
\sigma^{21}\left(Y_{, 1}^{1}, Y_{, 1}^{2}\right)
\end{array}\right) \quad[\nabla \sigma]=\left(\begin{array}{ll}
\sigma_{, 1}^{11} & \sigma_{, 2}^{11} \\
\sigma_{, 1}^{21} & \sigma_{, 2}^{21}
\end{array}\right)
$$

where in this case $\sigma_{1}^{11}$ denotes differentiation with respect to $Y_{, 1}^{1}$ and $\sigma_{.2}^{11}$ with respect to $Y_{, 1}^{2}$. With these notations, we have

$$
F^{\prime}(\Psi)=\left(\begin{array}{ccccc}
0 & 1 & 0 & 0 & 0 \\
-\frac{\left(\phi_{1}\right)^{2}}{\rho^{2}} & \frac{2 \phi_{1}}{\rho} & 0 & -\sigma_{, 1}^{11} & -\sigma_{, 2}^{11} \\
-\frac{\phi_{1} \phi_{2}}{\rho^{2}} & \frac{\phi_{2}}{\rho} & \frac{\phi_{1}}{\rho} & -\sigma_{, 1}^{21} & -\sigma_{, 2}^{21} \\
-\frac{\phi_{1} Y_{, 1}^{1}}{\rho^{2}} & \frac{Y_{1}^{1}}{\rho} & 0 & \frac{\phi_{1}}{\rho} & 0 \\
-\frac{\phi_{1} Y_{, 1}^{2}+\phi_{2}}{\rho^{2}} & \frac{Y_{1}^{2}}{\rho} & \frac{1}{\rho} & 0 & \frac{\phi_{1}}{\rho}
\end{array}\right)
$$

Taking into account (11) and (12), we have

$$
[\nabla \sigma][\nabla Y]=\left(\begin{array}{ll}
\sigma_{, 1}^{11} Y_{, 1}^{1}+\sigma_{, 2}^{11} Y_{, 1}^{2} & \sigma_{, 2}^{11} \\
\sigma_{, 1}^{21} Y_{, 1}^{1}+\sigma_{, 2}^{21} Y_{, 1}^{2} & \sigma_{, 2}^{21}
\end{array}\right)
$$

and hence

$\operatorname{Tr}([\nabla \sigma][\nabla Y])=\sigma_{, 2}^{21}+\sigma_{, 1}^{11} Y_{, 1}^{1}+\sigma_{, 2}^{11} Y_{, 1}^{2} \quad \operatorname{det}([\nabla \sigma][\nabla Y])=Y_{, 1}^{1}\left(\sigma_{, 1}^{11} \sigma_{, 2}^{21}-\sigma_{, 2}^{11} \sigma_{, 1}^{21}\right)$.

Denoting $\left(\alpha_{1}, \alpha_{2}\right)$ the eigenvalues of $-[\nabla \sigma][\nabla Y]$, the characteristic polynomial of this matrix is

$$
P(\lambda)=\left(\lambda-\alpha_{1}\right)\left(\lambda-\alpha_{2}\right)=\lambda^{2}+\operatorname{Tr}([\nabla \sigma][\nabla Y]) \lambda+\operatorname{det}([\nabla \sigma][\nabla Y])
$$

and therefore

$$
\begin{aligned}
& \alpha_{1}=\frac{-\operatorname{Tr}([\nabla \sigma][\nabla Y])+\sqrt{(\operatorname{Tr}([\nabla \sigma][\nabla Y]))^{2}-4 \operatorname{det}([\nabla \sigma][\nabla Y])}}{2} \\
& \alpha_{2}=\frac{-\operatorname{Tr}([\nabla \sigma][\nabla Y])-\sqrt{(\operatorname{Tr}([\nabla \sigma][\nabla Y]))^{2}-4 \operatorname{det}([\nabla \sigma][\nabla Y])}}{2}
\end{aligned}
$$

Finally, the eigenvalues of $F^{\prime}(\Psi)$ can be written under the form

$$
\Lambda^{E}=\left\{u_{1}, u_{1}+\sqrt{\frac{\alpha_{1}}{\rho}}, u_{1}-\sqrt{\frac{\alpha_{1}}{\rho}}, u_{1}+\sqrt{\frac{\alpha_{2}}{\rho}}, u_{1}-\sqrt{\frac{\alpha_{2}}{\rho}}\right\}
$$

and the conditions for the system to be hyperbolic are $\alpha_{1}>0$ and $\alpha_{2}>0$. Similar relationships can be determined for the lagrangian formulation. With an appropriate choice of $W$ well known models are obtained. 


\subsection{Examples of genuinely one-dimensional models}

In the following examples $u_{2}=0$ and $W_{\text {iso }}=0$.

\subsubsection{Compressible isotherm Euler equations}

Let $W_{\text {vol }}(J)=-\left(c_{0}\right)^{2} \ln (J)$ and $c_{0} \in \mathbb{R}$. We have

$$
\sigma\left(Y_{, 1}^{1}\right)=-\left(c_{0}\right)^{2} Y_{, 1}^{1} \quad \sigma^{\prime}\left(Y_{, 1}^{1}\right)=-\left(c_{0}\right)^{2}
$$

and the eulerian model reduces to

$$
(\rho u)_{t}+\left(\rho u_{1}^{2}+\left(c_{0}\right)^{2} Y_{, 1}^{1}\right)_{, 1}=0
$$

and $\rho=\rho_{0} Y_{, 1}^{1}$. Taking $\rho_{0}=1$, the characteristic speeds are

$$
\Lambda^{E}=\left\{u, u+c_{0}, u-c_{0}\right\}
$$

\subsubsection{Shallow water equations}

In this case $W_{\text {vol }}(J)=\frac{\beta}{J}$ and $\beta \in \mathbb{R}^{+}$. We have

$$
\sigma\left(Y_{, 1}^{1}\right)=-\frac{\beta}{2}\left(Y_{, 1}^{1}\right)^{2} \quad \sigma^{\prime}\left(Y_{, 1}^{1}\right)=-\beta Y_{, 1}^{1}
$$

and the eulerian model reduces to

$$
(\rho u)_{t}+\left(\rho u^{2}+\frac{\beta}{2}\left(Y_{, 1}^{1}\right)^{2}\right)_{, 1}=0
$$

and as before the continuity $\rho=\rho_{0} Y_{1}^{1}$. The characteristic speeds are

$$
\Lambda^{E}=\left\{u, u+\sqrt{\beta Y_{, 1}^{1}}, u-\sqrt{\beta Y_{, 1}^{1}}\right\}
$$

with $\rho_{0}=1$.

\subsubsection{Compressible stiffened gas}

A model for stiff fluids is obtained by taking an energy function that includes two different effects (see for example [10], [8], [6]). As for a gas, the energy function accounts for the phenomenon that an isoentropic compression induces an increase of internal energy. In contrast, an isoentropic expansion leads to a decrease of internal energy. This is of course fine for a gas, but if we consider a fluid or solid material where intermolecular forces are present, contrary to a gas, energy must increase when the density is reduced. The following energy functions accounts for these effects

$$
\varepsilon=\frac{W_{v o l}}{\rho_{0}}=\frac{\exp \left(\frac{s-s_{0}}{c_{v}}\right) \rho^{\gamma-1}}{\gamma-1}+\frac{p_{\infty}}{\rho}
$$

where $s_{0}$ is the reference entropy and $c_{v}, \gamma, p_{\infty} \in \mathbb{R}^{+}$are constants that characterize a given material. Other energy functions can be envisaged to 
mitigate or accentuate certain aspects of the resulting non-linear relation between stress and deformation. Using the above defined internal energy we find $p+p_{\infty}=\exp \left(\frac{s-s_{0}}{c_{v}}\right) \rho^{\gamma}$. The characteristic speeds are

$$
\Lambda^{E}=\left\{u, u+\sqrt{\gamma \frac{p+p_{\infty}}{\rho}}, u-\sqrt{\gamma \frac{p+p_{\infty}}{\rho}}\right\} ;
$$

if $p_{\infty}=0$ we recover the usual expressions for the compressible Euler equations.

\subsection{One-dimensional case with shear}

In two dimensions we define $\bar{B}=\frac{B}{\operatorname{det}(B)^{\frac{1}{2}}}$, so that $\operatorname{det}(\bar{B})=1$. Let now

$$
\varepsilon=\frac{W_{v o l}+W_{\text {iso }}}{\rho_{0}}=\frac{\exp \left(\frac{s-s_{0}}{c_{v}}\right) \rho^{\gamma-1}}{\gamma-1}+\frac{p_{\infty}}{\rho}+\frac{\chi}{\rho_{0}}(\operatorname{Tr}(\bar{B})-2)
$$

where $\chi \in \mathbb{R}^{+}$. The elastic part of the model (Neo-Hookean solid) accounts for elastic deformations in the transverse direction, i.e., $\sigma^{21} \neq 0$. We have in this case

$$
\begin{aligned}
\sigma^{11} & =p_{\infty}-\chi\left(\left(Y_{, 1}^{1}\right)^{2}+\left(Y_{, 1}^{2}\right)^{2}-1\right)-\kappa\left(Y_{, 1}^{1} \rho_{0}\right)^{\gamma} \\
\sigma^{12} & =-2 \chi Y_{, 1}^{2}
\end{aligned}
$$

and

$$
\begin{aligned}
\alpha_{1}= & +\frac{1}{2} \sqrt{\left(2 \chi\left(\left(Y_{, 1}^{1}\right)^{2}+\left(Y_{, 1}^{2}\right)^{2}+1\right)+\gamma \kappa\left(Y_{, 1}^{1} \rho_{0}\right)^{\gamma}\right)^{2}-8 \chi\left(2\left(Y_{, 1}^{1}\right)^{2} \chi+\gamma \kappa\left(Y_{, 1}^{1} \rho_{0}\right)^{\gamma}\right)} \\
& +\chi\left(\left(Y_{, 1}^{1}\right)^{2}+\left(Y_{, 1}^{2}\right)^{2}+1\right)+\frac{\gamma \kappa}{2}\left(Y_{, 1}^{1} \rho_{0}\right)^{\gamma} \\
\alpha_{2}= & -\frac{1}{2} \sqrt{\left(2 \chi\left(\left(Y_{, 1}^{1}\right)^{2}+\left(Y_{, 1}^{2}\right)^{2}+1\right)+\gamma \kappa\left(Y_{, 1}^{1} \rho_{0}\right)^{\gamma}\right)^{2}-8 \chi\left(2\left(Y_{, 1}^{1}\right)^{2} \chi+\gamma \kappa\left(Y_{, 1}^{1} \rho_{0}\right)^{\gamma}\right)} \\
& +\chi\left(\left(Y_{, 1}^{1}\right)^{2}+\left(Y_{, 1}^{2}\right)^{2}+1\right)+\frac{\gamma \kappa}{2}\left(Y_{, 1}^{1} \rho_{0}\right)^{\gamma}
\end{aligned}
$$

where $\kappa=\exp \left(\frac{s-s_{0}}{c_{v}}\right)$. Thanks to these expressions, the characteristic speeds are obtained from (13).

\section{Multimaterial solver}

In this section we summarize the steps of a classical finite-volume integration scheme for conservation laws and detail the sharp interface solver that we propose. We consider a one-dimensional problem since multidimensional schemes are often based on a dimensional splitting. Also, we focus on a first order explicit scheme in space and time. 
We assume that the initial condition at time $t_{n}$, the $n$-th time step, is known. Let $\Psi_{k}^{n}=\Psi\left(x_{k}, t_{n}\right)$, with $x_{k}$ the spatial coordinate $x$ of grid point $k$. The discretization points are $N+1$ and let $I=\{1, \cdots, N\}$. Consider two nonmiscible materials separated by a physical interface located, at time $t_{n}$, in $x_{f}^{n}$ and let $\iota=i$ such that $x_{i} \leq x_{f}<x_{i+1}, i \in I$. The space and time discretization $\forall k \in I$ and $k \neq \iota, \iota+1$ is as follows

$$
\frac{\Psi_{k}^{n+1}-\Psi_{k}^{n}}{\Delta t}=-\frac{\mathcal{F}_{k+1 / 2}^{n}\left(\Psi_{k}^{n}, \Psi_{k+1}^{n}\right)-\mathcal{F}_{k-1 / 2}^{n}\left(\Psi_{k-1}^{n}, \Psi_{k}^{n}\right)}{\Delta x}
$$

where $\Delta t=t^{n+1}-t^{n}, \Delta x=x_{k+1 / 2}-x_{k-1 / 2}$ and $\mathcal{F}_{k \pm 1 / 2}^{n}$ are the numerical fluxes evaluated at the cell interface located at $x_{k \pm 1 / 2}$. For consistency $\mathcal{F}$ is a regular enough function of both arguments and $\mathcal{F}(\Psi, \Psi)=F(\Psi)$. Numerical conservation requires that $\mathcal{F}\left(\Psi^{\prime}, \Psi\right)=\mathcal{F}\left(\Psi, \Psi^{\prime}\right)$.

The numerical flux function $\mathcal{F}_{k+1 / 2}\left(\Psi_{k}^{n}, \Psi_{k+1}^{n}\right)$ is computed either by an exact or by an approximate Riemann solver. In the following we use either Osher [13] or HLLC [15] approximate solvers. In any case, we assume that the Riemann solver employed defines at least two intermediate states $\Psi_{-}^{n}$ and $\Psi_{+}^{n}$, in addition to $\Psi_{k}^{n}$ and $\Psi_{k+1}^{n+1}$ and a contact discontinuity of speed $\left(u_{1}^{*}\right)^{n}$. The fluid speed is always continuous across the states $\Psi_{-}^{n}$ and $\Psi_{+}^{n}$. These states are defined so that mechanical equilibrium is ensured at the contact discontinuity. Let us assume that $\Psi_{-}^{n}$ is the state to the left of the contact discontinuity and $\Psi_{+}^{n}$ to the right.

The main idea is to use a standard numerical flux function $\mathcal{F}\left(\Psi_{k}, \Psi_{k+1}\right)$, $\forall k \in I, k \neq \iota$ and (16) to deduce $\Psi_{k}^{n+1}$. In contrast, for $\Psi_{\iota}^{n+1}$ and $\Psi_{\iota+1}^{n+1}$ we have

$$
\left\{\begin{array}{c}
\frac{\Psi_{\iota}^{n+1}-\Psi_{\iota}^{n}}{\Delta t}=-\frac{\mathcal{F}_{-}^{n}\left(\Psi_{-}^{n}\right)-\mathcal{F}_{\iota-1 / 2}^{n}\left(\Psi_{\iota-1}^{n}, \Psi_{\iota}^{n}\right)}{\Delta x} \\
\frac{\Psi_{\iota+1}^{n+1}-\Psi_{\iota+1}^{n}}{\Delta t}=-\frac{\mathcal{F}_{\iota+3 / 2}^{n}\left(\Psi_{\iota+1}^{n}, \Psi_{\iota+2}^{n}\right)-\mathcal{F}_{+}^{n}\left(\Psi_{+}^{n}\right)}{\Delta x}
\end{array}\right.
$$

where $\mathcal{F}_{ \pm}^{n}=F\left(\Psi_{ \pm}^{n}\right)$. The scheme is locally non conservative since $\mathcal{F}_{+}^{n} \neq \mathcal{F}_{-}^{n}$. However, the error in conservation is negligible as the shock speeds are predicted correctly in the numerical tests shown in Sec. 5 .

The interface position is updated in time using $\left(u_{1}^{*}\right)^{n}$, i.e., $x_{f}^{n+1}=x_{f}^{n}+$ $\left(u_{1}^{*}\right)^{n} \Delta t$. For numerical stability, the integration step is limited by the fastest of the characteristics over the grid points. Hence, the interface position will belong to the same interval between two grid points for more than one time step. When the physical interface overcomes a grid point, i.e., $x_{f}^{n+1} \geq x_{i+1}$ or $x_{f}^{n+1}<x_{i}$ then $\iota=i \pm 1$ accordingly. In other words, the above integration scheme is simply shifted of one point to the right or to the left. When the interface crosses a grid point, however, the corresponding conservative variables $\Psi_{\iota}^{n+1}$ do not correspond anymore to the material present at that grid point before the integration step. When $\iota=i+1$, i.e., the physical interface moves to the right of $i+1$, then we take $\Psi_{\iota}^{n+1}=\Psi_{-}^{n}$, whereas if $\iota=i-1, \Psi_{\iota}^{n+1}=\Psi_{+}^{n}$. It is noteworthy that the scheme presented here is reminiscent of that proposed in 3 for multifluids, starting from different considerations. 


\section{$5 \quad$ Numerical examples}

\subsection{Fluid - fluid interface}

We initially focus on test cases where the energy does not depend on isochoric deformations. The model reduces to the canonical compressible Euler equation with state equation given by (14). The numerical flux function $\mathcal{F}$ is that of Osher [13. The velocity $u_{1}^{*}$, the states $\Psi_{-}$and $\Psi_{-}$, are found by an exact Riemann problem solution at the interface. All the classical test cases of Tab. 1 have been considered. The values of the relevant variables and parameters to the left and to the right of the interface are denoted by $l$ and $r$, respectively. The same resolution was kept for all test cases, as well as the highest CFL before numerical instability occur. Not all the cases of Tab. 1 are presented the following figures. However, for the cases not presented, results similar to those shown are found. Here we present the cases 3, 7, and 9. The numerical solution is contrasted the exact one for most of these cases. The predominant pattern of the error at the interface is known as the overheating problem, and it is typical of many classical schemes. It can be observed for example in the test case 3 . The interface between the left and right states is sharp in all cases.

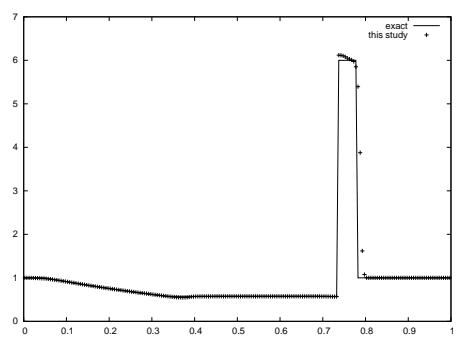

(a) density

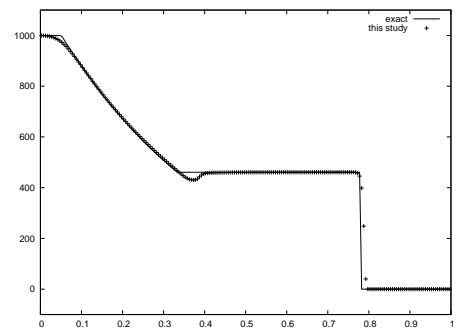

(c) pressure

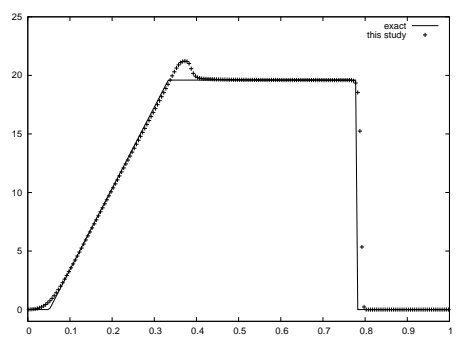

(b) velocity

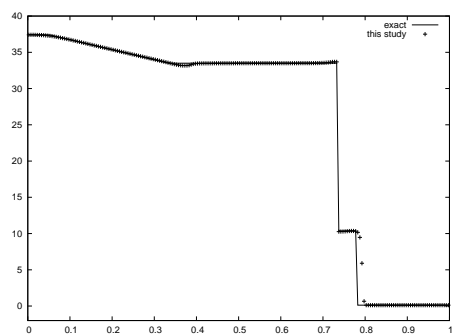

(d) speed of sound

Figure 1: Test 3 


\begin{tabular}{|c|c|c|c|c|c|c|c|c|c|c|c|c|c|c|}
\hline case & $\rho_{l}$ & $u_{l}$ & $p_{l}$ & $\gamma_{l}$ & $p_{\infty l}$ & $\rho_{r}$ & $u_{r}$ & $p_{r}$ & $\gamma_{r}$ & $p_{\infty r}$ & $x_{f}$ & $C F L$ & $N$ & $T_{\text {end }}$ \\
\hline 1 & 1.0 & 0.75 & 1.0 & 1.4 & 0.0 & 0.125 & 0 & 0.1 & 1.4 & 0.0 & 0.3 & 0.9 & 200 & 0.2 \\
\hline 2 & 1.0 & -2.0 & 4.0 & 1.4 & 0.0 & 1.0 & 2.0 & 4.0 & 1.4 & 0.0 & 0.5 & 0.9 & 200 & 0.15 \\
\hline 3 & 1.0 & 0.0 & 1000.0 & 1.4 & 0.0 & 1.0 & 0.0 & 0.01 & 1.4 & 0.0 & 0.3 & 0.5 & 200 & 0.012 \\
\hline 4 & 5.99924 & 19.5975 & 460.894 & 1.4 & 0.0 & 5.99924 & -6.19633 & 46.095 & 1.4 & 0.0 & 0.4 & 0.8 & 200 & 0.035 \\
\hline 5 & 0.1 & 1.0 & 1.0 & 1.4 & 0.0 & 1.0 & 1.0 .0 & 1.0 & 1.6 & 0.0 & 0.5 & 0.9 & 200 & 0.1 \\
\hline 6 & 1.0 & 0.0 & 1.0 & 1.4 & 0.0 & 0.125 & 0.0 & 0.1 & 1.6 & 0.0 & 0.5 & 0.9 & 200 & 0.1 \\
\hline 7 & 1.0 & 0.0 & 500.0 & 1.4 & 0.0 & 1.0 & 0.0 & 0.2 & 1.6 & 0.0 & 0.5 & 0.9 & 200 & 0.1 \\
\hline 8 & 3.984 & 27.355 & 1000.0 & 1.667 & 0.0 & 0.1 & 0.0 & 1.0 & 1.667 & 0.0 & 0.2 & 0.9 & 200 & 0.01 \\
\hline 9 & 1000.0 & 0.0 & $1.0 \mathrm{e} 9$ & 4.4 & $6.0 \mathrm{e} 8$ & 50.0 & 0.0 & $1.0 \mathrm{e} 5$ & 1.4 & 0.0 & 0.7 & 0.3 & 200 & 0.00024 \\
\hline
\end{tabular}

Table 1: Test cases. 


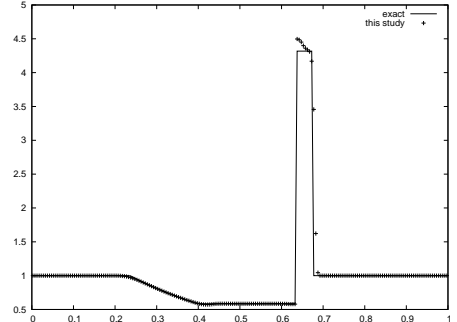

(a) density

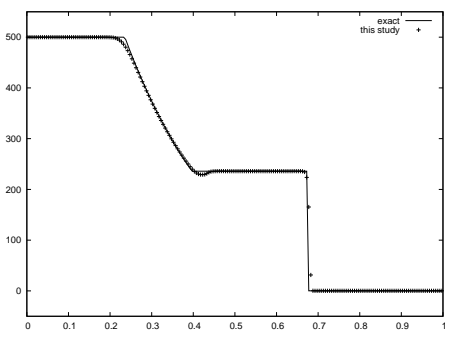

(c) pressure

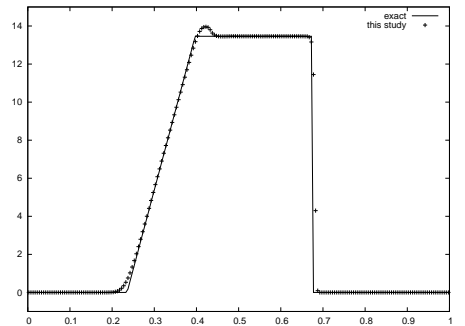

(b) velocity

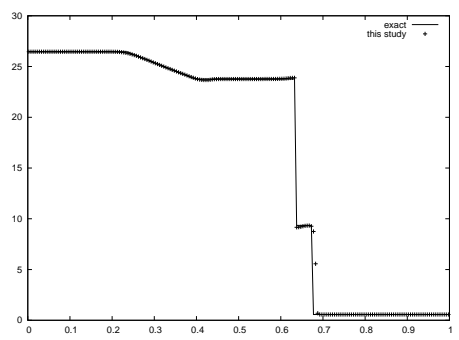

(d) speed of sound

Figure 2: Test 7

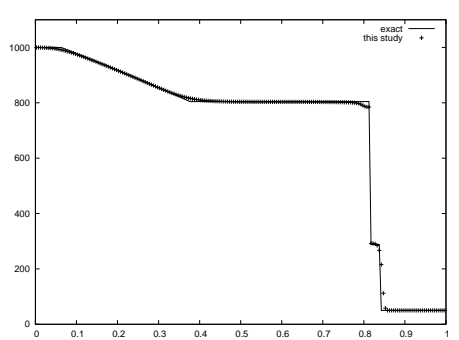

(a) density

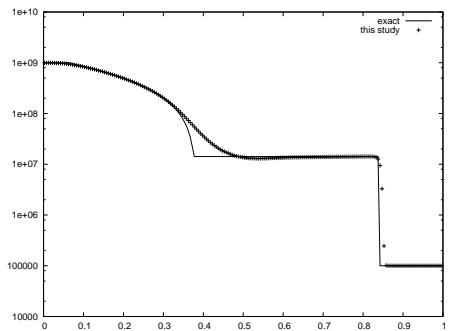

(c) pressure (log scale)

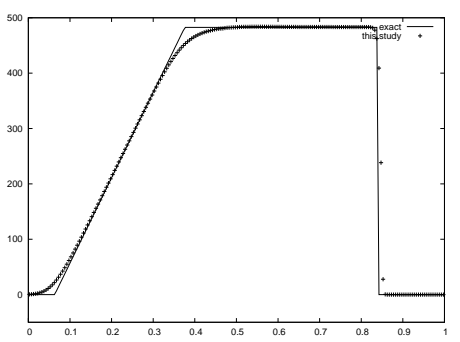

(b) velocity

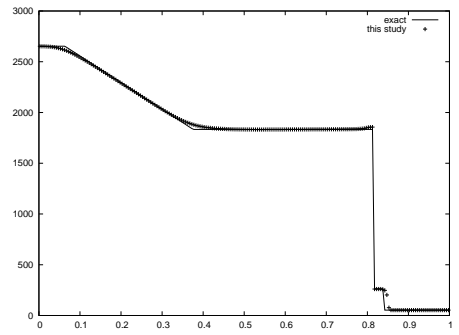

(d) speed of sound

Figure 3: Test 9 


\subsection{Solid - solid and solid - fluid interface}

The subsequent test cases are relevant to elastic materials with different physical properties separated by an interface. The configurations studied are similar to those in [6], but the constitutive law for the elastic model is different. Here we consider a Neo-Hookean material with energy expressed by (15). The density of the elastic material before deformation is constant, hence $\rho$ and $Y_{1}^{1}$ are proportional. The configurations under scrutiny are one-dimensional with non-zero transverse velocity. This velocity is constant in the transverse direction but may be variable in the longitudinal direction. Based on this hypothesis, the model used for the numerical simulations is

$$
\Psi=\left(\begin{array}{c}
\rho \\
\phi_{1} \\
\phi_{2} \\
Y_{, 1}^{2}
\end{array}\right) \quad F(\Psi)=\left(\begin{array}{c}
\phi_{1} \\
\frac{\left(\phi_{1}\right)^{2}}{\rho}-\sigma^{11} \\
\frac{\phi_{1} \phi_{2}}{\rho}-\sigma^{21} \\
\frac{\phi_{1} Y_{, 1}^{2}+\phi_{2}}{\rho} .
\end{array}\right)
$$

The numerical flux functions are determined based on the solution of the HLLC [15] approximate Riemann problem, similarly to what is done in [6]. Even though the exact wave pattern involves five waves (the fifth wave for the above model is brought back by the contact discontinuity), the approximate solver approaches the solution using three waves and thus defining only two intermediate states $\Psi_{-}$and $\Psi_{+}$. These states are the ones that define the numerical flux at the grid interface closest to the physical interface. More in details let $l$ and $r$ denote as before the states to the left and right and to the right of the cell interface concerned. Let $s_{r}$ and $s_{l}$ be the fastest rightward and leftward waves among those in (13). We have $s_{r}=\left(u_{1}+\sqrt{\frac{\alpha_{1}}{\rho}}\right)_{r}$ and $s_{l}=\left(u_{1}-\sqrt{\frac{\alpha_{1}}{\rho}}\right)$. We define also $Q_{r}=F\left(\Psi_{r}\right)-s_{r} \Psi_{r}, Q_{l}=F\left(\Psi_{l}\right)-s_{l} \Psi_{l}, Q_{-}=F\left(\Psi_{-}\right)-u_{1}^{*} \Psi_{-}$ and $Q_{+}=F\left(\Psi_{+}\right)-u_{1}^{*} \Psi_{+}$. The HLLC scheme is based on the assumption that $Q_{l}=Q_{-}, Q_{r}=Q_{+}$and $Q_{-}=Q_{+}$. From these equations, and relaxing the functional relation between $\sigma$ and $Y$ for the intermediate states $\Psi_{-}$and $\Psi_{+}$, we obtain

$$
\begin{aligned}
u_{1}^{*} & =\frac{Q_{l}^{2}-Q_{r}^{2}}{Q_{l}^{1}-Q_{r}^{1}} & u_{2}^{*} & =\frac{Q_{l}^{3}-Q_{r}^{3}}{Q_{l}^{1}-Q_{r}^{1}} \\
\left(\sigma^{11}\right)^{*} & =\frac{Q_{l}^{2} Q_{r}^{1}-Q_{r}^{2} Q_{l}^{1}}{Q_{l}^{1}-Q_{r}^{1}} & \left(\sigma^{12}\right)^{*} & =\frac{Q_{l}^{3} Q_{r}^{1}-Q_{r}^{3} Q_{l}^{1}}{Q_{l}^{1}-Q_{r}^{1}} \\
\rho_{-} & =\frac{Q_{l}^{1}}{u_{1}^{*}-s_{l}} & \rho_{+} & =\frac{Q_{r}^{1}}{u_{1}^{*}-s_{r}} \\
\left(Y_{, 1}^{2}\right)_{-} & =\frac{Q_{l}^{4}-u_{2}^{*}}{u_{1}^{*}-s_{l}} & \left(Y_{, 1}^{2}\right)_{+} & =\frac{Q_{r}^{4}-u_{2}^{*}}{u_{1}^{*}-s_{r}}
\end{aligned}
$$

where both velocity components and the the stress tensor are continuous across the contact discontinuity.

Particular care is needed when the interface separates an elastic material from a fluid. In that case $\sigma^{21}$ vanishes at the interface and therefore the transverse velocity is discontinuous. In this case the intermediate states $\Psi_{-}$and $\Psi_{+}$ 
are defined by

$$
\begin{array}{rlrl}
u_{1}^{*} & =\frac{Q_{l}^{2}-Q_{r}^{2}}{Q_{l}^{1}-Q_{r}^{1}} & \\
\left(\sigma^{11}\right)^{*} & =\frac{Q_{l}^{2} Q_{r}^{1}-Q_{r}^{2} Q_{l}^{1}}{Q_{l}^{1}-Q_{r}^{1}} & \left(\sigma^{21}\right)^{*}=0 & \rho_{+}=\frac{Q_{r}^{1}}{u_{1}^{*}-s_{r}} \\
\rho_{-} & =\frac{Q_{l}^{1}}{u_{1}^{*}-s_{l}} & \left(u_{2}\right)_{+}=\frac{Q_{r}^{3}}{\rho_{+}\left(u_{1}^{*}-s_{r}\right)} \\
\left(u_{2}\right)_{-} & =\frac{Q_{l}^{3}}{\rho_{-}\left(u_{1}^{*}-s_{l}\right)} & \left(Y_{, 1}^{2}\right)_{+}=\frac{Q_{r}^{4}-\left(u_{2}\right)_{-}}{u_{1}^{*}-s_{r}} &
\end{array}
$$

where now $\left(u_{2}\right)_{-}$and $\left(u_{2}\right)_{+}$are the transverse components of the velocity relative to the intermediate states $\Psi_{-}$and $\Psi_{+}$.

As an first example we consider a shock tube with solid copper initially at rest in the high pressure chamber $\left(10^{10} \mathrm{~Pa}\right)$ to the left of the interface initially located in $x_{f}=0.7 \mathrm{~m}$. The physical characteristics of copper, identical for all the test cases hereafter, are $p_{\infty}=342 \cdot 10^{8} \mathrm{~Pa}, \gamma=4.22, \chi=9.2 \cdot 10^{10} \mathrm{~Pa}$ and initial density $\rho_{0}=8.9 \cdot 10^{3} \mathrm{~kg} / \mathrm{m}^{3}$. In the low pressure chamber to the right of the interface there is air with initial $\rho_{0}=1 \mathrm{~kg} / \mathrm{m}^{3}$ and pressure $10^{5} \mathrm{~Pa}$. The grid is of 2000 points and the CFL is 0.8 . Since the transverse velocity is uniform, only three waves are present in the field (Fig. 4(a)): an expansion fan travelling in copper to the left, a shock wave propagating in air to the right, and the contact discontinuity. The density and the pressure are discontinuous at the interface and the interface is sharp (Fig. 4(a) and (d)). The normal stress and the horizontal speed are continuous at the interface (Fig. 4(b) and (c)). The logarithmic scale for $p$ and $\sigma^{11}$ is necessary to put in evidence the shock wave. 


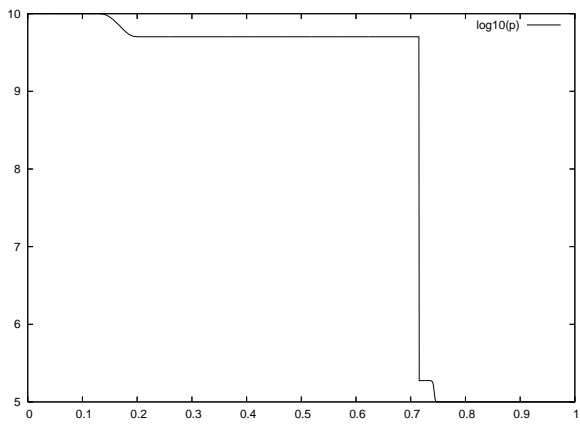

(a) $p$

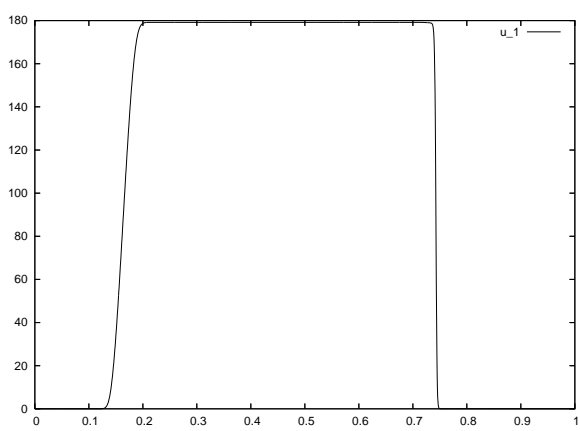

(c) $u_{1}$

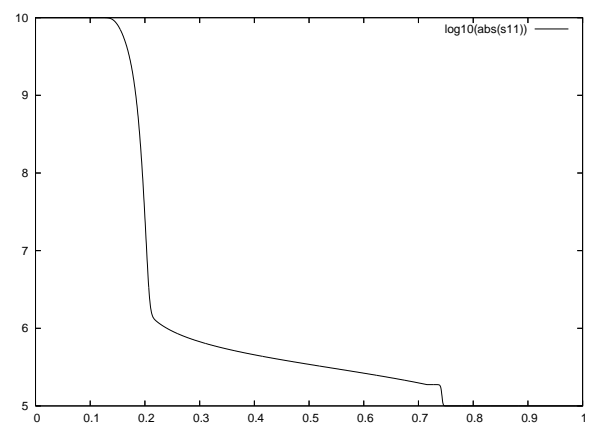

(b) $\sigma^{11}$

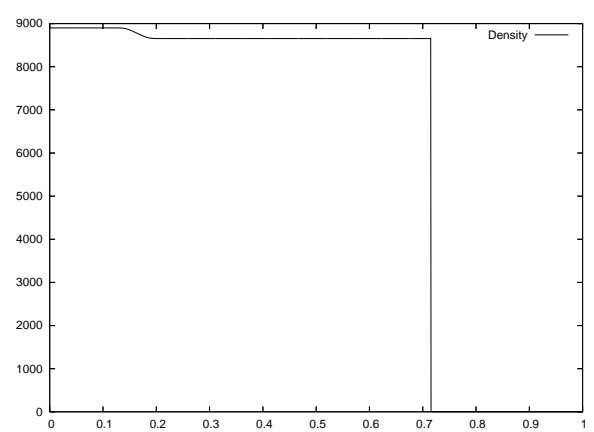

(d) $\rho$

Figure 4: Solid - air shock tube with high pressure and density ratios; $t=$ $8.42 \times 10^{-5}$ s. $x_{1}$ on the horizontal axis.

The next case (see Fig. 5) is a solid - solid interface with shear. We consider a shock tube filled with copper whose density is initially $\rho_{0}=8.9 \cdot 10^{3} \mathrm{~kg} / \mathrm{m}^{3}$. An interface separates the high pressure chamber on the left where the copper is at rest with pressure $10^{8} \mathrm{~Pa}$ and the same material on the right at low pressure $\left(10^{5} \mathrm{~Pa}\right)$. This time a tangential velocity discontinuity is imposed: $u_{2}=0 \mathrm{~m} / \mathrm{s}$ on the left and $u_{2}=100 \mathrm{~m} / \mathrm{s}$ on the right. The interface is initially located at $x_{f}=0.5 \mathrm{~m}$. The grid is of 2000 points and the CFL is 0.8 . Five waves corresponding to a couple of shear stress waves, two normal stress waves and the contact discontinuity are present in the field. The fastest waves are those relative to normal stress. The shear and the normal stress, the horizontal and the vertical speeds are continuous at the interface (Fig. 5(c), (d), (e), (f)). The density and the pressure are discontinuous at the interface and the interface is sharp (Fig. 5(a) and (b)). Although the material constitutive law is different, the results are in good qualitative accordance to those presented in [6]. 


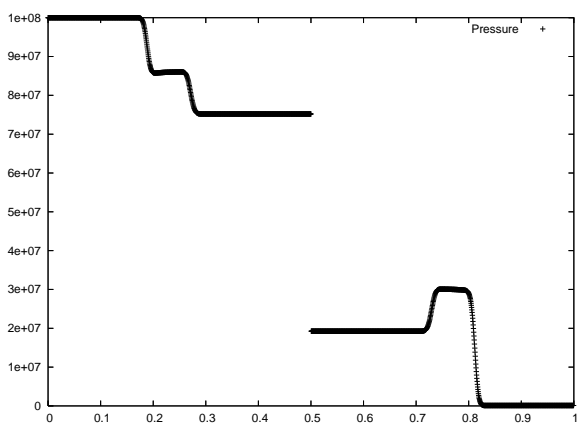

(a) $p$

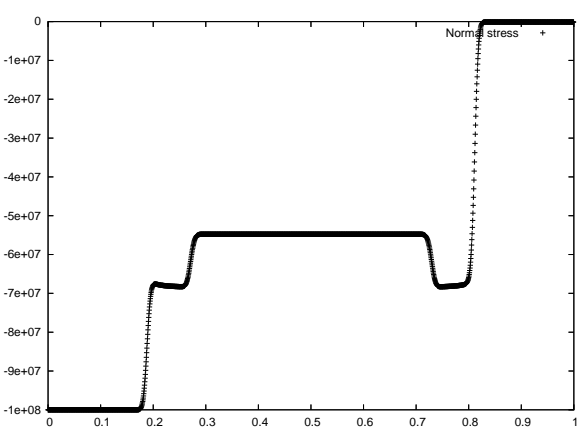

(c) $\sigma^{11}$

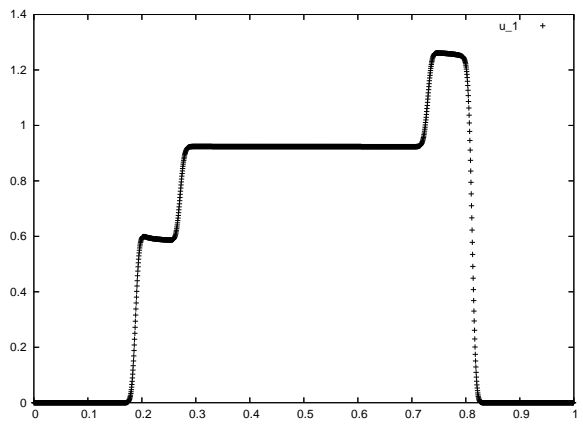

(e) $u_{1}$

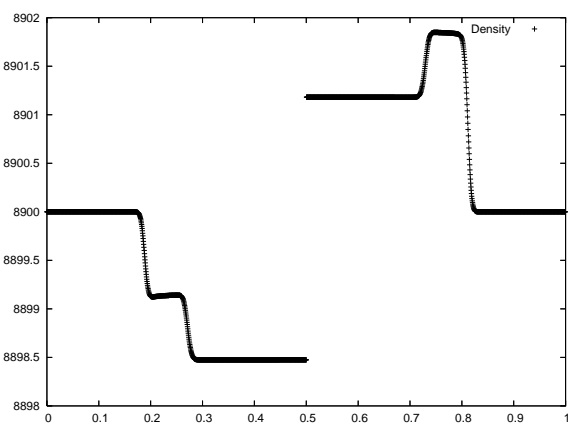

(b) $\rho$

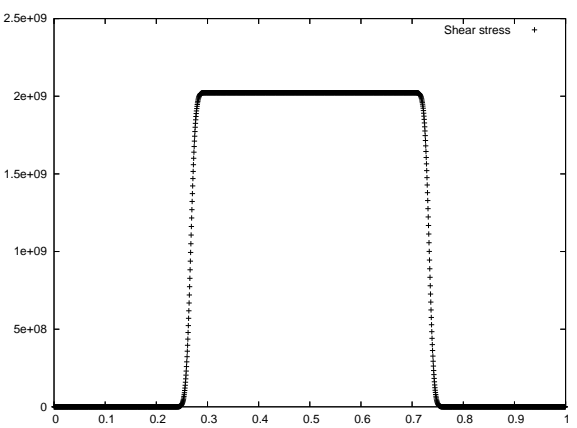

(d) $\sigma^{12}$

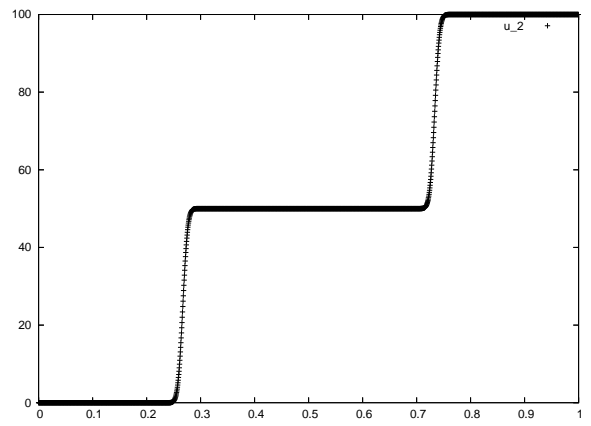

(f) $u_{2}$

Figure 5: Solid solid interface with shear; $t=5.13 \times 10^{-5} \mathrm{~s} . x_{1}$ on the horizontal axis.

The final test case concerns a copper-air interface with discontinuous initial conditions in the copper. The copper-air interface is at $x_{1}=0.4 \mathrm{~m}$. To the left of the interface there is copper with initially $\rho_{0}=8.9 \cdot 10^{3}$. To the right there is air with initially $\rho_{0}=1 \mathrm{~kg} / \mathrm{m}^{3}$. The initial conditions are uniform static pressure $\left(10^{5} \mathrm{~Pa}\right)$ and uniform horizontal velocity $(0)$ across the materials. Inside copper the vertical velocity is $10^{3} \mathrm{~m} / \mathrm{s}$ between $x_{1}=0$ and $x_{1}=0.15 \mathrm{~m}$ and 0 elsewhere. The vertical velocity in air is 0 . The left boundary conditions are such that the solution is symmetric. The results are presented in Fig 6 and Fig 7 . The initial discontinuity in vertical velocity in copper at time 0 breaks down in two waves 
travelling in opposite directions. These waves are reflected on the left border and on the copper-air interface, giving rise to subsequent wave interactions. Although the material constitutive laws are different, the results are in good qualitative accordance to those presented in [6]. 


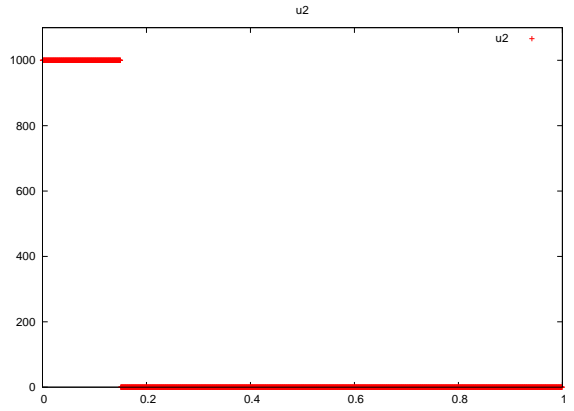

(a) $t=0 \mathrm{~s}$

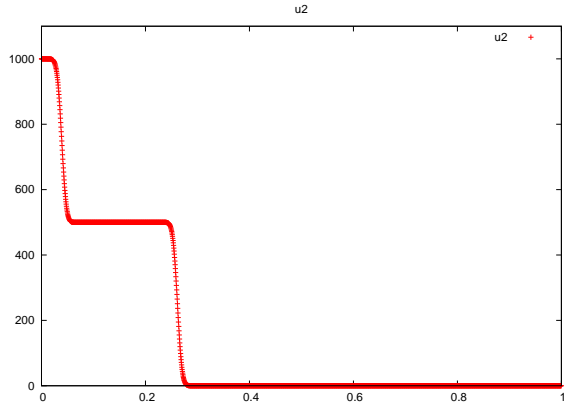

(c) $t=2.4 \times 10^{-5} \mathrm{~s}$

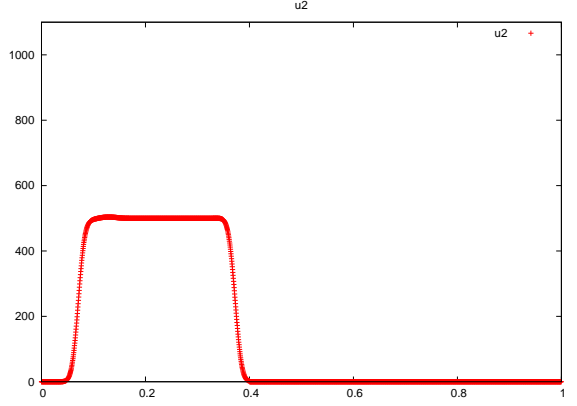

(e) $t=4.8 \times 10^{-5} \mathrm{~s}$

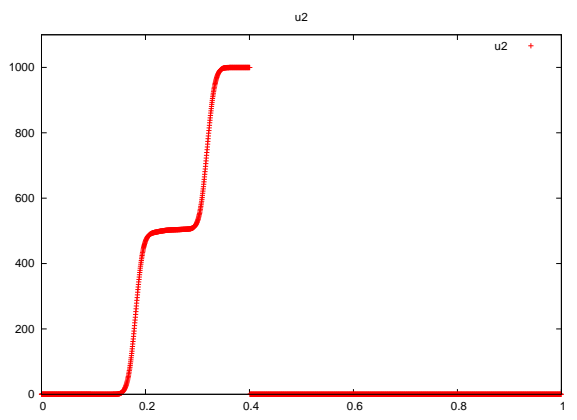

(g) $t=7.3 \times 10^{-5} \mathrm{~s}$

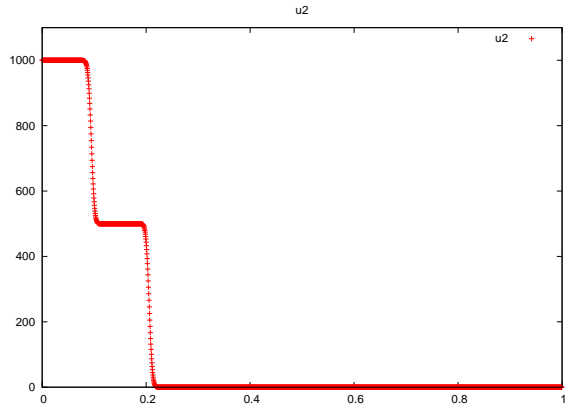

(b) $t=1.2 \times 10^{-5} \mathrm{~s}$

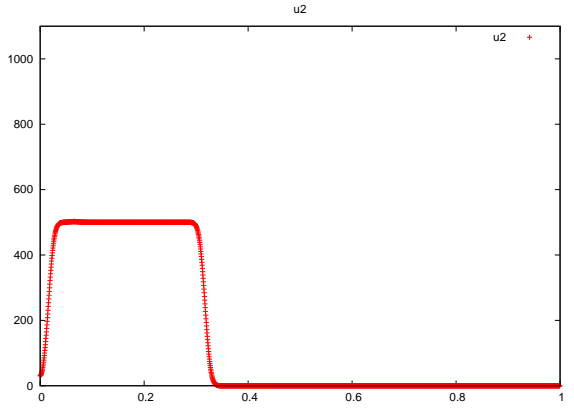

(d) $t=3.6 \times 10^{-5} \mathrm{~s}$

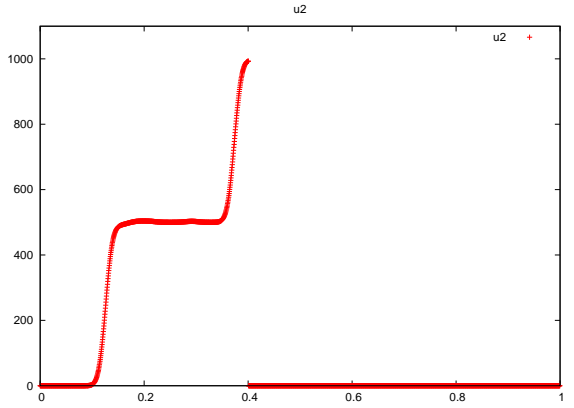

(f) $t=6.1 \times 10^{-5} \mathrm{~s}$

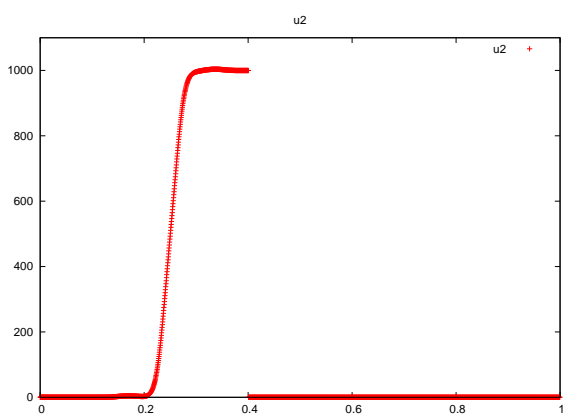

(h) $t=8.5 \times 10^{-5} \mathrm{~s}$

Figure 6: Copper-air interface with discontinuous initial conditions in the copper. Transverse velocity $u_{2}$. 


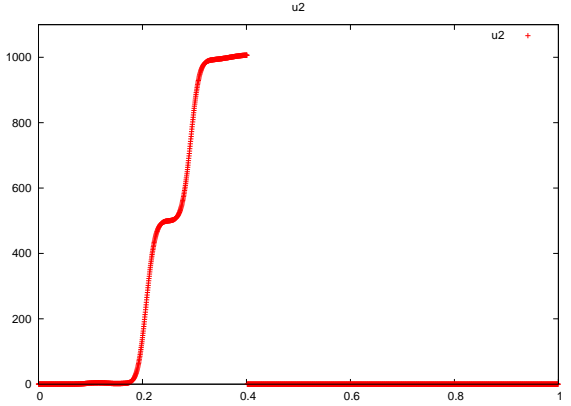

(a) $t=9.7 \times 10^{-5} \mathrm{~s}$

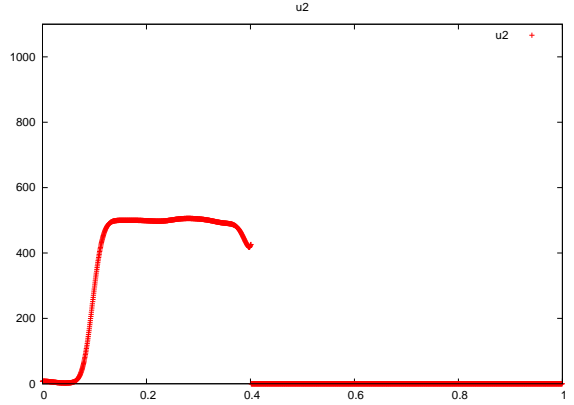

(c) $t=12.2 \times 10^{-5} \mathrm{~s}$

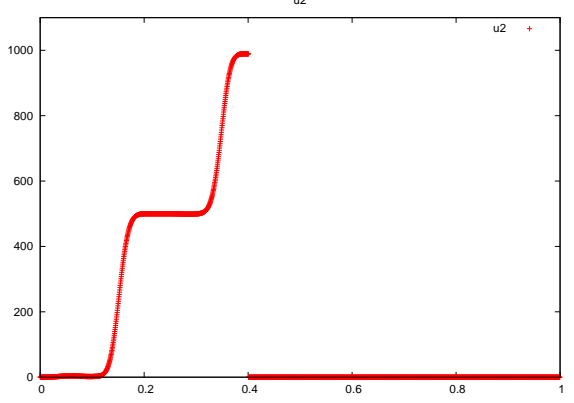

(b) $t=10.1 \times 10^{-5} \mathrm{~s}$

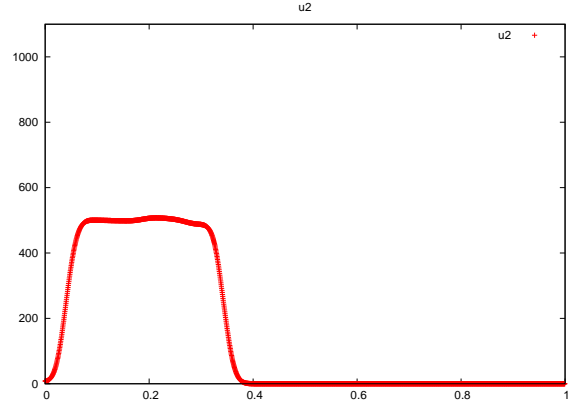

(d) $t=13.4 \times 10^{-5} \mathrm{~s}$

Figure 7: Copper-air interface with discontinuous initial conditions in the copper. Transverse velocity $u_{2}$.

\section{Conclusions}

We presented a simple method to deal with multimaterial interfaces. This method is based on a locally non-conservative scheme. The results show that the contact discontinuity is sharp and that the numerical solution is generally in good agreement with the exact one or with numerical solutions presented in the literature, even when large velocity, pressure and density differences characterize the flow. Extensions to multidimensional configurations and to higher accuracy seem attainable by classical means and are under current investigation.

\section{References}

[1] R. Abgrall. How to prevent pressure oscillations in multicomponent flow calculations: a quasi-conservative approach. J Comput Phys, 125:150-160, 1996.

[2] R. Abgrall and S. Karni. Computations of compressible multifluids. J Comput Phys, 169(2):594-623, 2001.

[3] A. Chertock, S. Karni, and A. Kurganov. Interface tracking method for compressible multifluids. M2AN, 42:991-1019, 2008. 
[4] P.G. Ciarlet. Mathematical elasticity Vol I, Three dimensional elasticity. Volume 20 of Studies in Mathematics and its Applications, 1994.

[5] G.-H. Cottet, E. Maitre, and T. Milcent. Eulerian formulation and level set models for incompressible fluid-structure interaction. M2AN, 42:471-492, 2008.

[6] N. Favrie, S.L. Gavrilyuk, and R. Saurel. Solid-fluid diffuse interface model in cases of extreme deformations. J Comput Phys, 228(16):6037-6077, 2009.

[7] S. Galerab, P.H. Maire, and J. Breilb. A two-dimensional unstructured cell-centered multi-material ale scheme using vof interface reconstruction. J Comput Phys, 229(16):5755-5787, 2010.

[8] S.L. Gavrilyuk, N. Favrie, and R. Saurel. Modelling wave dynamics of compressible elastic materials. J Comput Phys, 227(5):2941-2969, 2008.

[9] S.K. Godunov. Elements of continuum mechanics. Nauka Moscow, 1978.

[10] X.Y. Hu, N.A. Adams, and G. Iaccarino. On the hllc riemann solver for interface interaction in compressible multi-fluid flow. J Comput Phys, 228:6572-6589, 2009.

[11] G. Kluth and B. Després. Discretization of hyperelasticity on unstructured mesh with a cell-centered lagrangian scheme. J Comput Phys, 229(24):9092-9118, 2010.

[12] G.H. Miller and P. Colella. A high-order eulerian godunov method for elastic-plastic flow in solids. J Comput Phys, 167(1):131-176, 2001.

[13] S. Osher and S. Chakravarthy. Upwind schemes and boundary conditions with applications to euler equations in general geometries. J Comput Phys, 50:447-481, 1983.

[14] H. Terashima and G. Tryggvason. A front-tracking method with projected interface conditions for compressible multi-fluid flows. Comput Fluids, 39:1804-1814, 2010.

[15] E.F. Toro, M. Spruce, and W. Speares. Restoration of the contact surface in the hll-riemann solver. Shock Waves, 4:25-34, 1994. 
Centre de recherche INRIA Bordeaux - Sud Ouest

Domaine Universitaire - 351, cours de la Libération - 33405 Talence Cedex (France)

Centre de recherche INRIA Grenoble - Rhône-Alpes : 655, avenue de l'Europe - 38334 Montbonnot Saint-Ismier

Centre de recherche INRIA Lille - Nord Europe : Parc Scientifique de la Haute Borne - 40, avenue Halley - 59650 Villeneuve d'Ascq

Centre de recherche INRIA Nancy - Grand Est : LORIA, Technopôle de Nancy-Brabois - Campus scientifique 615, rue du Jardin Botanique - BP 101 - 54602 Villers-lès-Nancy Cedex

Centre de recherche INRIA Paris - Rocquencourt : Domaine de Voluceau - Rocquencourt - BP 105 - 78153 Le Chesnay Cedex

Centre de recherche INRIA Rennes - Bretagne Atlantique : IRISA, Campus universitaire de Beaulieu - 35042 Rennes Cedex

Centre de recherche INRIA Saclay - Île-de-France : Parc Orsay Université - ZAC des Vignes : 4, rue Jacques Monod - 91893 Orsay Cedex

Centre de recherche INRIA Sophia Antipolis - Méditerranée : 2004, route des Lucioles - BP 93 - 06902 Sophia Antipolis Cedex 